\title{
Global tuberculosis prevention: should we start from the beginning?
}

\author{
Giovanni Sotgiu (1) ${ }^{1}$, Delia Goletti ${ }^{2}$ and Alberto Matteelli ${ }^{3}$
}

Affiliations: ${ }^{1}$ Clinical Epidemiology and Medical Statistics Unit, Dept of Medical, Surgical and Experimental Sciences, University of Sassari, Sassari, Italy. ${ }^{2}$ Translational Research Unit, National Institute for Infectious Diseases, Rome, Italy. ${ }^{3}$ Institute of Infectious and Tropical Diseases, WHO Collaborating Centre for TB/HIV coinfection and TB elimination, University of Brescia, Brescia, Italy.

Correspondence: Giovanni Sotgiu, Clinical Epidemiology and Medical Statistics Unit, Dept of Medical, Surgical and Experimental Sciences, University of Sassari, Via Padre Manzella, 4, Sassari - 07100, Italy. E-mail: gsotgiuduniss.it

@ERSpublications

TB prevention should be based on a global and local comprehensive assessment of LTBI burden http://bit.ly/2YfKM0T

Cite this article as: Sotgiu G, Goletti D, Matteelli A. Global tuberculosis prevention: should we start from the beginning?. Eur Respir J 2019; 54: 1901394 [https://doi.org/10.1183/13993003.01394-2019].

Tuberculosis (TB), which is estimated to be the deadliest infectious disease worldwide with $\sim 1.6$ million deaths yearly [1], is a clinical issue due to the "ancient" diagnostics and drugs currently available. If compared with the management of other highly prevalent infectious diseases, such as HIV/AIDS and hepatitis $\mathrm{C}$ virus infection, it is clear that the clinical approach for TB disease requires innovation, being still based on means with limited efficacy (e.g. treatment of multidrug-resistant TB; MDR-TB), and characterised by the denominator "long duration": conventional bacteriological diagnosis, therapy for the drug-susceptible disease (i.e. 6 months) and MDR-TB (i.e. $>20$ months) [2-7].

The slow declining annual global TB incidence rate $(\sim 2 \%)$ is partially explained by the above-mentioned limitations in terms of diagnostic and therapeutic tools. The determinants of the current epidemiological scenario include the epidemic of co-infected TB/HIV cases and the emergence and spread of MDR-TB [8, 9].

The cascade of care shows several shortcomings and pitfalls, not only at peripheral healthcare centres in low income and high TB incidence countries, but also in TB reference centres located in high income countries $[10,11]$.

The infectiousness of pulmonary Mycobacterium tuberculosis cases has still important uncertainties. Patients with culture-confirmed pulmonary disease can transmit mycobacterial strains to susceptible individuals, determining secondary infection and then, eventually, the disease. The mismanagement of the contacts of contagious TB cases and poor infection control measures implemented in healthcare centres and the communities play a significant role in the persistence of the pool of infected individuals who can develop the disease. The exposure to contagious cases and the occurrence of infection depend on several factors linked to the host, mycobacterial characteristics and environment, which may interplay, thereby increasing or decreasing the risk of infection. Unfortunately, those risk factors are only partly known and, therefore, interventions aimed at neutralising the effect of exposure cannot be comprehensive $[12,13]$.

Several modelling studies have shown that if countries want to eliminate the economic and health burden (i.e. annual TB incidence rate $<1$ case per million) of TB, it is important to have a policy of disease control which implies the appropriate clinical management of the patients and their contacts; however, disease 
control measures may not be enough. More efforts are needed to reduce exposure (e.g. improved and well-implemented infection control policies), to protect susceptible individuals (e.g. primary prevention based on an effective and safe new vaccine administered to neonates and at highest risk population groups in high and low TB incidence countries, respectively), and to detect and treat individuals with a latent TB infection (LTBI) $[8,9]$.

The World Health Organization (WHO) stresses the potential key role of the above-mentioned public health interventions by including them in the End TB Strategy approved by the World Health Assembly in May 2014. In particular, the programmatic management of LTBI was recognised by the global healthcare community as one of the crucial public health interventions needed to decrease TB incidence $[8,9]$.

Currently, individuals at high risk of developing active TB should undergo diagnostic work-up to detect M. tuberculosis infection using indirect, old-fashioned (i.e. tuberculin skin test;TST) and new (interferon- $\gamma$ release assays; IGRAs) immunological tools. However, their diagnostic accuracy, which has been evaluated in those with active TB, as a surrogate for sensitivity, is below the expected standards. In particular, both TST and IGRAs have a low sensitivity in immunocompromised patients, such as those with HIV infection or those exposed to biological therapy. In addition, TST has a low specificity in individuals immunised with BCG vaccine or with non-tuberculous mycobacterial infections [14-16].

Preventive therapy has been suggested by WHO as a key intervention measure to achieve TB elimination [17]. The burden of LTBI worldwide was estimated in 2016 at 1.7 billion cases, that is, a quarter of the global population $[18,19]$. Owing to the safety and tolerability of the currently available LTBI treatment regimens, as well as their long duration, preventive therapy currently targets only high-risk populations, meaning the objective of eradication is hardly within reach. Still, screening of individuals at the highest risk of developing $\mathrm{TB}$, and consequent preventive therapy in those scored positive, appear the smartest strategies to reduce the burden of LTBI and, therefore, the rate of TB incident cases $[7,8,17]$.

The WHO guidelines for the treatment of LTBI, issued in 2015 and published in the European Respiratory Journal, clearly outline the framework of processes and outputs expected in more than 100 high and high/ middle income countries, where the TB incidence is lower than 100 cases per 100000 population, after the adoption and adaptation of evidence-based recommendations focused on LTBI diagnosis and therapy among vulnerable populations $[17,20]$.

Participants at the United Nations High-Level Meeting on TB, held in New York in September 2018, highlighted the importance of LTBI diagnosis and therapy and the clear identification of the suitable population target $[21,22]$.

According to the most recent WHO reports, the implementation and scale-up of the guidelines was suboptimal at a national and local level in several European and non-European countries [1, 23].

To better address the challenge of the LTBI elimination and to adequately assess the healthcare, economic, and financial impact of the LTBI strategy it is key to better estimate the number of latently infected individuals that should be diagnosed and treated.

The manuscript of CoHEN et al. [24], entitled "The global prevalence of latent tuberculosis: a systematic review and meta-analysis", published in this issue of the European Respiratory Journal, describes LTBI prevalence worldwide. Different from previous studies that used mathematical models, they systematically collated the current evidence published in scientific journals and provided quantitative estimates with a meta-analysis approach. They adopted an innovative method of estimation based on the computation of LTBI cases using both TST and/or IGRAs (both QuantiFERON and T-SPOT.TB). They selected studies enrolling >200 individuals, to avoid selection bias, and carried out since 2005, when IGRA methodology was deemed reliable. Moreover, they excluded studies focused only on specific population groups (e.g. those with HIV) which could hinder the interpretation and the inference of their findings. A total of 88 studies, representing 36 high and low TB incidence countries, were selected: 67 studies used TST and 41 IGRAs.

The global LTBI prevalence ranged from $24.8 \%$ (IGRAs) to $21.2 \%$ (TST $>10 \mathrm{~mm}$ ) and strongly correlated ( $\mathrm{r} \sim 0.7$ ) with TB disease incidence. Regarding the analysis performed on IGRAs, different estimates of prevalence were found: if the indeterminate results were included in those scored positive or negative, the sensitivity for LTBI prevalence slightly changed from $26.3 \%$ to $24.2 \%$, respectively. Similarly, if TST different cut-offs were used (i.e. 5, 10 and $15 \mathrm{~mm}$ ), the LTBI prevalence changed from $24.1 \%, 21.2 \%$, and $17.4 \%$ respectively.

The analysis combining TST and IGRAs found a prevalence of LTBI of 25.2\% (IGRAs-based prevalence) and $27.1 \%$ (>10 mm TST-based prevalence). Therefore, CoHEn et al. [24] in this study found global LTBI prevalence similar to that reported by HouBEN and DodD [19] in 2016, with an estimate of 23\%, but lower 
compared with that $32 \%$ estimated by WHO in 1999. The higher LTBI prevalence estimated by WHO can be attributed to the epidemiology of 1999 , when TB incidence was higher with consequently higher $M$. tuberculosis transmission rates.

Interestingly, IGRA-based studies found a higher LTBI prevalence in comparison with TST-based surveys. However, studies based on both IGRAs and TST tests recruiting a pooled population of 43861 (IGRAs) and 44238 (TST) found a comparable prevalence of $25.2 \%$ (excluding indeterminate results) and $27.1 \%$ (TST cut-off $10 \mathrm{~mm}$ ), respectively.

The findings of this study on global LTBI prevalence are key for the strategy based on reliable targets. Although methodological shortcomings can be detected (e.g. selection of specific geographical settings, convenience sampling, low specificity of TST, high between-study heterogeneity), a strong message from this systematic review can be delivered: the LTBI prevalence is approximately one quarter of the global population and, then, similar to the estimate proposed by Houben and DoDD [19], but lower than that estimated by WHO more than 20 years ago. Southeast Asia and Africa are the WHO regions with the highest prevalence needing an immediate intervention and a regional strategy. Governmental and non-governmental associations, as well as international and national scientific societies, should consider changing strategic TB approaches that are prone to leaving LTBI unaddressed. Critical discussion is strongly recommended, as is scientific support, pending continuous economic and logistical support.

Great efforts are needed to discover new diagnostic tools that can predict the risk of TB disease, as currently available tests (TST and IGRAs) are poorly predictive $[16,25]$. In the comparative studies using both TST and IGRAs, similar LTBI prevalence was found. This needs further confirmation in comparative population-based surveys. This indirect information on diagnostic accuracy could be important in the discussion on the best tools to adopt in LTBI surveys.

Last but not least, surveillance/survey programmes should be tailored also on LTBI at a national and local level $[18,19,24,26,27]$. The implementation of the WHO guidelines and their impact should be quantitatively measured with indicators. Therefore, new plans aimed at describing the epidemiological scenario and the effectiveness of public health interventions are urgently needed [28]. The methodological approach suggested by the study of COHEN et al. [24] could be implemented in single studies and can be helpful in the monitoring process of one of the key components of the WHO End TB Strategy.

Conflict of interest: G. Sotgiu has nothing to disclose. D. Goletti reports grants and personal fees for consultancy from Quidel, personal fees for lectures from Qiagen, Diasorin and Jensen, personal fees for consultancy from Biomerieux, outside the submitted work. A. Matteelli has nothing to disclose.

\section{References}

1 World Health Organization. Global Tuberculosis Report 2018. Geneva, World Health Organization, 2018. www. who.int/tb/publications/global_report/en/

2 Pai M, Sotgiu G. Diagnostics for latent TB infection: incremental, not transformative progress. Eur Respir J 2016; 47: 704-706.

3 Lewinsohn DM, Leonard MK, LoBue PA, et al. Official American Thoracic Society/Infectious Diseases Society of America/Centers for Disease Control and Prevention Clinical Practice Guidelines: diagnosis of tuberculosis in adults and children. Clin Infect Dis 2017; 64: 111-115.

4 Nahid P, Dorman SE, Alipanah N, et al. Official American Thoracic Society/Centers for Disease Control and Prevention/Infectious Diseases Society of America Clinical Practice Guidelines: treatment of drug-susceptible tuberculosis. Clin Infect Dis 2016; 63: e147-e195.

5 Sotgiu G, Nahid P, Loddenkemper R, et al. The ERS-endorsed official ATS/CDC/IDSA clinical practice guidelines on treatment of drug-susceptible tuberculosis. Eur Respir J 2016; 48: 963-971.

6 Migliori GB, Sotgiu G, Rosales-Klintz S, et al. ERS/ECDC Statement: European Union standards for tuberculosis care, 2017 update. Eur Respir J 2018; 51: 1702678.

7 Collaborative Group for the Meta-Analysis of Individual Patient Data in MDR-TB treatment-2017, Ahmad N, Ahuja SD, et al. Treatment correlates of successful outcomes in pulmonary multidrug-resistant tuberculosis: an individual patient data meta-analysis. Lancet 2018; 392: 821-834.

8 Lönnroth K, Migliori GB, Abubakar I, et al. Towards tuberculosis elimination: an action framework for low-incidence countries. Eur Respir J 2015; 45: 928-952.

9 D’Ambrosio L, Dara M, Tadolini M, et al. Tuberculosis elimination: theory and practice in Europe. Eur Respir J 2014; 43: 1410-1420.

10 Migliori GB, Sotgiu G, D'Ambrosio L, et al. TB and MDR/XDR-TB in European Union and European Economic Area countries: managed or mismanaged? Eur Respir J 2012; 39: 619-625.

11 Sotgiu G, D'Ambrosio L, Centis R, et al. TB and M/XDR-TB infection control in European TB reference centres: the Achilles' heel? Eur Respir J 2011; 38: 1221-1223.

12 Dye C, Bassili A, Bierrenbach AL, et al. Measuring tuberculosis burden, trends, and the impact of control programmes. Lancet Infect Dis 2008; 8: 233-243.

13 Rieder HL. The dynamics of tuberculosis epidemiology. Indian J Tuberc 2014; 61: 19-29.

14 Pai M, Denkinger CM, Kik S V, et al. Gamma interferon release assays for detection of Mycobacterium tuberculosis infection. Clin Microbiol Rev 2014; 27: 3-20. 
15 Diel R, Goletti D, Ferrara G, et al. Interferon- $\gamma$ release assays for the diagnosis of latent Mycobacterium tuberculosis infection: a systematic review and meta-analysis. Eur Respir J 2011; 37: 88-99.

16 Petruccioli E, Scriba TJ, Petrone L, et al. Correlates of tuberculosis risk: predictive biomarkers for progression to active tuberculosis. Eur Respir J 2016; 48: 1751-1763.

17 Getahun H, Matteelli A, Abubakar I, et al. Management of latent Mycobacterium tuberculosis infection: WHO guidelines for low tuberculosis burden countries. Eur Respir J 2015; 46: 1563-1576.

18 Dye C, Scheele S, Dolin P, et al. Consensus statement. Global burden of tuberculosis: estimated incidence, prevalence, and mortality by country. WHO Global Surveillance and Monitoring Project. JAMA 1999; 282: 677-686.

19 Houben RM, Dodd PJ. The global burden of latent tuberculosis infection: a re-estimation using mathematical modelling. PLoS Med 2016; 13: e1002152.

20 World Health Organization. Latent Tuberculosis Infection: Updated and Consolidated Guidelines for Programmatic Management. Geneva, World Health Organization, 2018.

21 Sakamoto H, Lee S, Ishizuka A, et al. Challenges and opportunities for eliminating tuberculosis - leveraging political momentum of the UN high-level meeting on tuberculosis. BMC Public Health 2019; $19: 76$.

22 Herbert N, Sharma V, Masham BS, et al. Concrete action now: UN High-Level Meeting on Tuberculosis. Lancet Infect Dis 2018; 18: 709-710.

23 World Health Organization. Meeting of the Implementation Core Group of WHO Global Task Force on Latent TB Infection and Country Stakeholders on Implementation Tools and Joint TB and HIV Programming to Scale Up TB Preventive Treatment. Geneva, World Health Organization, 2018.

24 Cohen A, Mathiasen VD, Schön T, et al. The global prevalence of latent tuberculosis: a systematic review and meta-analysis. Eur Respir J 2019; 54: 1900655.

25 Gupta RK, Turner CT, Venturini C, et al. Concise whole blood transcriptional signatures for incipient tuberculosis: a systematic review and patient level pooled meta-analysis. BioRxiv 2019; preprint [https://doi.org/ $10.1101 / 668137]$

26 Knight GM, McQuaid CF, Dodd PJ, et al. Global burden of latent multidrug-resistant tuberculosis: trends and estimates based on mathematical modelling. Lancet Infect Dis 2019; 19: 903-912.

27 Garcia-Basteiro AL, Jenkins HE, Rangaka M. The burden of latent multidrug-resistant tuberculosis. Lancet Infect Dis 2019; 19: 802-803.

28 Churchyard GJ, Swindells S. Controlling latent TB tuberculosis infection in high-burden countries: A neglected strategy to end TB. PLoS Med 2019; 16: e1002787. 